Pacific Journal of Mathematics

GENERATORS FOR EVOLUTION SYSTEMS WITH QUA 


\title{
GENERATORS FOR EVOLUTION SYSTEMS WITH QUASI CONTINUOUS TRAJECTORIES
}

\author{
JAMES V. HEROD
}

With $G$ a normed space, this paper provides conditions on a nonlinear function $A$ from $R \times G$ to $G$ in order to insure that if $P$ is in $G$ then there will be a (not necessarily continuous) solution $Y$ for

$$
Y(x)=P+\int_{0}^{x} d_{t} A(t, Y(t)) .
$$

Early work in the study of the Stieltjes integral equation

$$
M(x, z)=1+\int_{x}^{z} d F M(I, z)
$$

was done by H. S. Wall [25] and T. H. Hildebrandt [8]. In Wall's paper, $F$ is a continuous matrix valued function which is of bounded variation on each finite interval. Hildebrandt dropped the requirement of continuity and used a modified Stieltjes integral. J. S. Mac Nerney carefully analysed these ideas in a series of papers which led to the fundamental relationships found in [15], [16], and [17].

The papers [15] and [17] establish two classes $O A$ and $O M$ of functions and a one-to-one pairing of the classes made possible through a continuously continued sum, a continuously continued product, and a Stieltjes integral equation. In [17], if $V$ is in $O A, M$ is in $O M, S$ is a linearly ordered set, and $P$ is contained in a complete, normed, Abelian group, then $V$ and $M$ are related by $M(x, y) P=$ ${ }_{x} \Pi^{y}[1+V] P, V(x, y) P={ }_{x} \Sigma^{y}[M-1] P$, and $M(x, y) P=P+\int_{x}^{y} V M(I, y) P$.

The results in [15] may be identified with analogous results in ordinary differential equations associated with nonautonomous, continuous, linear systems and [17] may be identified with Lipschitz systems. An indication of the nature of the generality obtained in the Stieltjes integral equation theory is found in [16], or in David L. Lovelady's discussion of interface problems [11, p. 184], or in a recent paper by Robert $H$. Martin [20] which investigates a linear operator equation and which identifies the linearly ordered set as the positive integers. Additional results related to [15] were found by B. W. Helton and Davis-Chatfield (see [2] or [3]). Also, this author determines a characterization of subsets of the two classes $O A$ and $O M$ which give rise to invertible evolution operators $M$ in [4], for the linear case, and in [7] for the nonlinear (but Lipschitz) case. 
In [9] Don Hinton and in [1] Carl Bitzer develop a theory for Stieltjes-Volterra equations. Reneke shows in [21] and [23] that much of the classical Volterra theory is contained in [15] or [17].

Questions concerning bounds for solutions of Stieltjes equations, as well as perturbations of these solutions have been investigated by Schamedeke and Sell [24], Herod [5], Martin [19], Reneke [22], and Lovelady [10], [11], and [12]. Also, Marrah and Proctor [18] have found results concerning periodic solutions.

In [6], this author extends the classes $O A$ and $O M$ by using some of the ideas of analytic semi-group theory. In that investigation, similar to Mac Nerney's, two classes $O A$ and $O M$ are paired by a continuously continued sum, a continuously continued product, and a Riemann-Stieltjes equation. (In this setting, also, Lovelady [14] has generalized earlier results of his involving perturbations of the systems.) The Lipschitz condition of [17] was dropped in [6] at the expense of requiring that $M(\cdot, y) P$, in addition to being of bounded variation on each finite interval, be continuous and that $S$ should be the real line. The results which follow relax these requirements.

We suppose that $S$ is a nondegenerate set with a linear ordering and that $\{S, \geqq\}$ has the least upper bound property. Also, $\{G,+,|\cdot|\}$ denotes a complete, normed Abelian group with zero element 0 . Further, suppose that $D$ is a closed subset of $G$ and that $V$ is a function such that if each of $x$ and $y$ is in $S$ and $x \geqq y$ then $V(x, y)$ is a function from $D$ into $G$ having the following properties:

(i) If $x \geqq y \geqq z$ and $P$ is in $D$ then $V(x, y) P+V(y, z) P=V(x, z) P$,

(ii) If $a>b$ then there is a nondecreasing, numerical valued function $\beta$ defined on $S$ such that if $\varepsilon>0$ and $P$ is in $D$ then there is a positive number $\delta$ having the property that if $Q$ is in $D$ such that $|Q-P|<\delta$ and $a \geqq x \geqq y \geqq b$ then $|V(x, y) P-V(x, y) Q| \leqq$ $[\beta(x)-\beta(y)] \varepsilon$,

(iii) If $a>b$ then $D$ is contained in the range of $[1-V(a, b)]$ and if $P$ and $Q$ are in $D$ then $|[1-V(a, b)] P-[1-V(a, b)] Q| \geqq$ $|P-Q|$, and

(iv) If $a>b$ and $P$ is in $D$ then there is a nondecreasing, numerical function $\alpha$ such that if $\left\{s_{p}\right\}_{0}^{n}$ is a nonincreasing sequence with values in $[b, a]$ and $a \geqq x \geqq y \geqq b$ then $\mid V(x, y) \prod_{p=1}^{n}[1-$ $\left.V\left(s_{p-1}, s_{p}\right)\right]^{-1} P \mid \leqq \alpha(x)-\alpha(y)$.

If $f$ is a function from $S$ with values in $G$ and $y$ is in $S$ then $f\left(y^{-}\right)$is a member $g$ of $G$ having the property that if $\varepsilon>0$ then there is a member $x$ of $S$ such that $x<y$ and if $x \leqq t<y$ then $|g-f(t)|<\varepsilon$. In a similar manner, $f\left(y^{+}\right)$may be defined.

The following theorems are established: 
Theorem I. If $a>b, \beta$ is as in (ii), $P$ is in $D$, and $\varepsilon>0$ then there is a subdivision $s$ of $\{a, b\}$ such that if $t$ is a refinement of $s$ then

$$
\left|\Pi_{s}[1-V]^{-1} P-\Pi_{t}[1-V]^{-1} P\right|<\{4+2[\beta(a)-\beta(b)]\} \varepsilon .
$$

Let $M$ be a function defined as follows: If $x \geqq y$ and $P$ is in $D$ then $M(x, y) P={ }_{x} \Pi^{y}[1-V]^{-1} P$.

Theorem II. If $a>b$ then $M(a, b)$ is a function from $D$ to $D$ and $|P-Q|$,

(1) If each of $P$ and $Q$ is in $D$ then $|M(a, b) P-M(a, b) Q| \leqq$

(2) If $x \geqq y \geqq z$ and $P$ is in $D$ then $M(x, y) M(y, z) P=M(x, z) P$,

(3) If $P$ is in $D$, and $a \geqq x \geqq y \geqq b$ then $|M(x, b) P-M(y, b) P| \leqq$ $\alpha(x)-\alpha(y)$,

(4) If $a \geqq b, \varepsilon>0$, and $P$ is in $D$ then there is a positive number $\delta$ having the property that if $Q$ is in $D$ such that $|Q-P|<\delta$ and $a \geqq x \geqq y \geqq b$ then $|[M(x, y)-1] P-[M(x, y)-1] Q| \leqq[\beta(x)-$ $\beta(y)] \varepsilon$.

Theorem III. If $P$ is in $D$ and $b$ is a member of $S$ then the only function $g$ which is of bounded variation on each finite interval of $S$ and which satisfies the integral equation $g(x)=P+(L) \int_{x}^{b} V[g]$ for each $x \geqq b$ is given by $g(x)=M(x, b) P$ for $x \geqq b$.

Proof of Theorem I.

Lemma 1. If $a>b, P$ is in $D$, and $\alpha$ is as in (iv), then

(1) $\lim _{x \downarrow b}\left([1-V(x, b)]^{-1} P\right)$ exists and is $\left[1-V\left(b^{+}, b\right)\right]^{-1} P$ and

(2) If $t$ is a subdivision of $\{a, b\}$ then $\mid \Pi_{t}[1-V]^{-1} P-$ $\left[1-V\left(b^{+}, b\right]^{-1} P \mid \leqq \alpha(a)-\alpha\left(b^{+}\right)\right.$,

(3) $\lim _{x \uparrow a}\left([1-V(a, x)]^{-1} P\right)$ exists and is $\left[1-V\left(a, a^{-}\right)\right]^{-1} P$ and

(4) If $t$ is a subdivision of $\{a, b\}$ then $\mid \Pi_{t}[1-V]^{-1} P-$ $\left[1-V\left(a, a^{-}\right)\right]^{-1} P \mid \leqq \alpha\left(a^{-}\right)-\alpha(b)$.

Indication of proof. Suppose that $x \geqq y>b$. Then

$$
\begin{aligned}
& \left|[1-V(x, b)]^{-1} P-[1-V(y, b)]^{-1} P\right| \\
\leqq & \left|V(x, b)[1-V(y, b)]^{-1} P-V(y, b)[1-V(y, b)]^{-1} P\right| \\
\leqq & \alpha(x)-\alpha(y) .
\end{aligned}
$$

The existence of $\lim _{x \downarrow b} \alpha(x)$, together with the fact that $D$ is closed, implies the existence of $\lim _{x \downarrow b}\left([1-V(x, b)]^{-1} P\right)$ in $D$. Let $Q$ be this 
limit. Then $|[1-V(x, b)] Q-P| \leqq\left|Q-[1-V(x, b)]^{-1} P\right|+\mid V(x, b) Q-$ $V(x, b)[1-V(x, b)]^{-1} P \mid$. Consequently, $P=\lim _{x \downarrow b}[1-V(x, b)] Q=$ $\left[1-V\left(b^{+}, b\right)\right] Q$. That is, $Q=\left[1-V\left(b^{+}, b\right)\right]^{-1} P$ so that $(1)$ is established. In order to establish (2), suppose that $\left\{t_{p}\right\}_{0}^{n}$ is a subdivision of $\{a, b\}$. With $Q$ as above,

$$
\begin{aligned}
\mid \prod_{p=1}^{n} & {\left[1-V\left(t_{p-1}, t_{p}\right)\right]^{-1} P-Q \mid } \\
& \leqq\left|\prod_{p=1}^{n}\left[1-V\left(t_{p-1}, t_{p}\right)\right]^{-1} P-\left[1-V\left(t_{n-1}, t_{n}\right)\right]^{-1} P\right|+\alpha\left(t_{n-1}\right)-\alpha\left(b^{+}\right) \\
& \leqq \sum_{p=1}^{n-1}\left|V\left(t_{p-1}, t_{p}\right)\left[1-V\left(t_{n-1}, t_{n}\right)\right]^{-1} P\right|+\alpha\left(t_{n-1}\right)-\alpha\left(b^{+}\right) \\
& \leqq \alpha(a)-\alpha\left(b^{+}\right) .
\end{aligned}
$$

In a similar manner, one can establish (3) and (4).

Lemma 2. Suppose that $a>b, \beta$ is as in (ii), $\varepsilon$ is a positive number, and $P$ is in $D$. There is a subdivision $\left\{s_{p_{0}}^{m}\right.$ of $\{a, b\}$ such that if $\left\{t_{p}\right\}_{0}^{n}$ is a refinement of $s$ and $k$ is a sequence such that $t\left(k_{p}\right)=s_{p}, p=0,1, \cdots, m$, then

$$
\begin{aligned}
\sum_{p=1}^{m} \sum_{q=1+k_{p-1}}^{k_{p}} \mid V\left(t_{q-1}, t_{q}\right) \prod_{i=q}^{k_{p}}\left[1-V\left(t_{\imath-1}, t_{i}\right)\right]^{-1} \prod_{j=p+1}^{m}\left[1-V\left(s_{j-1}, s_{j}\right)\right]^{-1} P \\
\quad-V\left(t_{q-1}, t_{q}\right) \prod_{i=1 \uparrow k_{p-1}}^{k_{p}}\left[1-V\left(t_{i-1}, t_{\imath}\right)\right]^{-1} \prod_{j=p+1}^{m}\left[1-V\left(s_{j-1}, s_{j}\right)\right]^{-1} P \mid \\
< \\
<[4+2(\beta(a)-\beta(b))] \varepsilon .
\end{aligned}
$$

Proof. With the supposition of the lemma, let $\alpha$ be as in (iv). Define functions $\Delta, \delta$, and $d$ as follows:

If $R$ is in $D$ then $A(R)$ is the largest number $e$ not exceeding 1 and having the property that if $Q$ is in $D,|Q-R|<e$, and $a \geqq$ $x \geqq y \geqq b$ then $|V(x, y) Q-V(x, y) R| \leqq[\beta(x)-\beta(y)] \varepsilon$,

If $b \leqq z<a, R$ is in $D$, and $Q=\lim _{x \downarrow z}[1-V(x, z)]^{-1} R$ then $\delta(z, R)$ is defined as follows: If there is no point $y$ such that $z<y<a$ then $\delta(z, R)=a$ and, otherwise, $\delta(z, R)$ is the least upper bound of all $x$ such that $z<x \leqq a$ and such that if $z \leqq y<x$ and $t$ is a subdivision of $\{y, z\}$ then $\left|\Pi_{t}[1-V]^{-1} R-Q\right|<\Delta(Q)$, and

If $b \leqq z<y \leqq a$ and $c$ is a positive number then let $a$ be the greatest lower-bound of all $w$ such that $z \leqq w$ and such that if $w \leqq u<y$ then $\alpha\left(y^{-}\right)-\alpha(u)<c$. If there is no point of $S$ between $x$ and $y$ let $d(y, z, c)$ be $x$. If there is, let $d(y, z, c)$ be such a point. Note that if $u$ is in $S$ and $d(y, z, c) \leqq u<y$ then $\alpha\left(y^{-}\right)-\alpha(u)<c$.

Define the sequence $u$ as follows: $u_{0}=b, u_{2}=\delta\left(u_{0}, P\right), u_{1}=$ $d\left(u_{2}, u_{0}, \varepsilon\right)$, and, if $n$ is a positive integer, 


$$
u_{2 n+2}=\delta\left(u_{2 n}, \prod_{q=1}^{2 n}\left[1-V\left(u_{2 n-q+1}, u_{2 n-q}\right)\right]^{-1} P\right)
$$

and $u_{2 n+1}=d\left(u_{2 n+2}, u_{2 n}, \varepsilon / 2^{n}\right)$. Assume that $u$ is an infinite sequence. Since $u$ is nondecreasing and bounded, let $u_{\infty}$ be $\lim u_{p}$ and, for each positive integer $j$, let $R_{j}=\prod_{q=1}^{j}\left[1-V\left(u_{j-q+1}, u_{j-q}\right)\right]^{-1} P$. If $m>n$ then, as in [6, p. 250] $\left|R_{m}-R_{n}\right| \leqq \alpha\left(u_{m}\right)-\alpha\left(u_{n}\right)$. Because $\lim _{x \uparrow u_{\infty}} \alpha(x)$ exists, $\left\{R_{p}\right\}_{p=1}^{\infty}$ converges. For each integer $n$, let $Q_{n}=\lim _{x \downarrow u_{n}}[1-$ $\left.V\left(x, u_{n}\right)\right]^{-1} R_{n}$. The sequence $\left\{Q_{p}\right\}_{p=1}^{\infty}$ converges for suppose that $\gamma$ is a positive number. Let $R_{\infty}=\lim R_{p}$ and let $v$ be a member of $S$ such that if $u_{\infty}>x \geqq v$ then $\alpha\left(x^{+}\right)-\alpha(x)<\gamma / 2$. Let $N$ be a positive integer such that if $n>N$ then $\left|R_{\infty}-R_{n}\right|<\gamma / 2$ and $u_{\infty}>u_{n} \geqq v$. Then $\lim Q_{p}=R_{\infty}$ for $\left|R_{\infty}-Q_{n}\right|<\alpha\left(u_{n}^{+}\right)-\alpha\left(u_{n}\right)+\gamma / 2$. By [6, Lemma 2.1] there is a positive number $\xi$ such that if $n$ is a positive integer then $\Delta\left(Q_{n}\right)>\xi$. Again, using the fact that $\lim _{x \uparrow u_{\infty}} \alpha(x)$ exists, there is an integer $N$ such that if $m>n>N$ then $\alpha\left(u_{m}\right)-$ $\alpha\left(u_{n}\right)<\xi$ and, in this case, if $t$ is a subdivision of $\left\{u_{m}, u_{n}\right\}$ then $\left|\Pi_{t}[1-V]^{-1} R_{n}-Q_{n}\right|<\alpha\left(u_{m}\right)-\alpha\left(u_{n}^{+}\right)<\xi \leqq \Delta\left(Q_{n}\right)$. Hence, $\delta\left(u_{n}, R_{n}\right) \geqq u_{m}$. Because this holds for each integer $m>n, \delta\left(u_{n}, R_{n}\right) \geqq u_{\infty}$. This is a contradiction to the assumption that $u$ is an infinite sequence.

Let $m$ be the least integer such that $u_{2 m}=a$, and define $s_{p}$ to be $u_{2 m-p}$ for $p=1,2, \cdots, 2 m$. Let $\left\{t_{q}\right\}_{q=0}^{n}$ be a refinement of $s$ and $k$ be an increasing sequence such that $k_{0}=0, k_{2 m}=n$, and $t\left(k_{p}\right)=s_{p}$ for $p=0,1, \cdots, 2 m$. If $p$ is an integer in $[1, m]$ and $q$ is an integer in $\left[1+k_{2 p-1}, k_{2 p}\right]$ then $u_{2(m-p)+2}=\delta\left(u_{2(m-p)}, R_{2(m-p)}\right)$. Hence

$$
\left|\prod_{i=q}^{k 2 p}\left[1-V\left(t_{i-1}, t_{i}\right)\right]^{-1} R_{2(m-p)}-Q_{2(m-p)}\right|<\Delta\left(Q_{2(m-p)}\right)
$$

and

$$
\begin{aligned}
& \left|V\left(t_{q-1}, t_{q}\right) \prod_{i=q}^{k_{2} p}\left[1-V\left(t_{i-1}, t_{i}\right)\right]^{-1} R_{2(m-p)}-V\left(t_{q-1}, t_{q}\right) Q_{2(m-p)}\right| \\
& \quad \leqq\left[\beta\left(t_{q-1}\right)-\beta\left(t_{q}\right)\right] \varepsilon .
\end{aligned}
$$

If $p$ is an integer in $[1, m]$ and $q$ is an integer in $\left[1+k_{2 p-2}, k_{2 p-1}\right]$ then

$$
\begin{aligned}
& \mid V\left(t_{q-1}, t_{q}\right) \prod_{i=q}^{k_{2} p-1}\left[1-V\left(t_{i-1}, t_{i}\right)\right]^{-1} \prod_{j=2 p}^{2 m}\left[1-V\left(s_{j-1}, s_{j}\right)\right]^{-1} P \\
& \quad-V\left(t_{q-1}, t_{q}\right) \prod_{i=1+k_{2 p-2}}^{k_{2 p-1}}\left[1-V\left(t_{i-1}, t_{i}\right)\right]^{-1} \prod_{j=2 p}^{2 m}\left[1-V\left(s_{j-1}, s_{j}\right)\right]^{-1} P \mid
\end{aligned}
$$

is zero if $q=1+k_{2 p_{-2}}$ and does not exceed $2\left[\alpha\left(t_{q-1}\right)-\alpha\left(t_{q}\right)\right]$ if $1+k_{2 p-2}<q \leqq k_{2 p-1}$. Furthermore, $\alpha\left(t_{k_{2 p-2}}\right)-\alpha\left(t_{k_{2 p-1}}\right)=\alpha\left(s_{2 p-2}\right)-$ $\alpha\left(s_{2 p-1}\right)=\alpha\left(u_{2(m-p)+2}^{-}\right)-\alpha\left(u_{2(m-p+1)}\right)<\varepsilon / 2^{m-p}$. It follows that 


$$
\begin{aligned}
& \sum_{p=1}^{2 m}\left\{\sum_{q=1+k_{p-1}}^{k_{p}} \mid V\left(t_{q-1}, t_{q}\right) \prod_{i=q}^{k_{p}}\left[1-V\left(t_{i-1}, t_{\imath}\right)\right]^{-1} \prod_{j=p+1}^{2 m}\left[1-V\left(s_{j-1}, s_{j}\right)\right]^{-1} P\right. \\
&\left.\quad-V\left(t_{q-1}, t_{q}\right) \prod_{i=1+k_{p-1}}^{k_{p}}\left[1-V\left(t_{i-1}, t_{i}\right)\right]^{-1} \prod_{j=p+1}^{2 m}\left[1-V\left(s_{j-1}, s_{j}\right)\right]^{-1} P \mid\right\} \\
&=\sum_{p=1}^{m}\left\{\sum_{q=1+k_{2 p-2}}^{k_{2 p-1}} \mid V\left(t_{q-1}, t_{q}\right) \prod_{i=q}^{k_{2 p-1}}\left[1-V\left(t_{2-1}, t_{2}\right)\right]^{-1} \prod_{j=2 p}^{2 m}\left[1-V\left(s_{j-1}, s_{j}\right)\right]^{-1} P\right. \\
& \quad-V\left(t_{q-1}, t_{q}\right) \prod_{i=1+k_{2 p}-2}^{k_{2 p-1}}\left[1-V\left(t_{i-1}, t_{i}\right)\right]^{-1} \prod_{j=2 p}^{2 m}\left[1-V\left(s_{j-1}, s_{j}\right)\right]^{-1} P \\
& \quad+\sum_{q=2+k_{2 p-1}}^{k_{2 p}} \mid V\left(t_{q-1}, t_{q}\right) \prod_{i=q}^{k_{2 p}}\left[1-V\left(t_{i-1}, t_{i}\right)\right]^{-1} R_{2(m-p)} \\
&\left.\left.\quad-V\left(t_{q-1}, t_{q}\right) \prod_{i=1}^{k_{2} p}\left[1-V\left(t_{i-1}, t_{\imath}\right)\right]^{-1} R_{2(m-p}\right) \mid\right\} \\
& \leqq \sum_{p=1}^{m} \varepsilon / 2^{m-p}+\sum_{p=1}^{m} 2\left[\beta\left(s_{2 p-1}-\right)-\beta\left(s_{2 p}\right)\right] \varepsilon<\{4+2[\beta(a)-\beta(b)]\} \varepsilon .
\end{aligned}
$$

Indication of proof for Theorem I. The inequalities in the proof of Theorem 2.1 on pages 251 and 252 of [6] carry over almost without change by using the above Lemma 2 .

The techniques above also provide the following

Corollary. If $a>b, \beta$ is as in (ii), $P$ is in $D$, and $\varepsilon>0$ then there is a subdivision $s$ of $\{a, b\}$ such that if $\left\{t_{p}\right\}_{0}^{n}$ is a refinement of $s$ and $p$ is an integer in $[0, n]$ then $\mid M\left(t_{p}, b\right) P-\prod_{i=p+1}^{n}[1-$ $\left.V\left(t_{\imath-1}, t_{\imath}\right)\right]^{-1} P \mid<\varepsilon$.

Proof of Theorem II. Parts (1) and (2) follow from the corresponding inequalities for the approximations to $M$; further details are indicated in Theorem 2.2 of [6]. To establish part 3 of Theorem II, suppose that $a \geqq x \geqq y \geqq b$ and $P$ is in $D$. Let $\alpha$ be as in (iv), and $t$ and $s$ be a subdivision of $\{x, y\}$ and $\{y, b\}$ respectively. Then

$$
\begin{aligned}
& |M(x, b) P-M(y, b) P| \leqq\left|M(x, b) P-\Pi_{t}[1-V]^{-1} \Pi_{s}[1-V]^{-1} P\right| \\
& \quad+\left|\left\{\Pi_{t}[1-V]^{-1}-1\right\} \Pi_{s}[1-V]^{-1} P\right| \\
& \quad+\left|\Pi_{s}[1-V]^{-1} P-M(y, b) P\right| .
\end{aligned}
$$

Also,

$$
\begin{aligned}
& \left|\left\{\prod_{t}[1-V]^{-1}-1\right\} \prod_{s}[1-V]^{-1} P\right| \\
& \quad=\left|\sum_{p=1}^{n} V\left(t_{p-1}, t_{p}\right) \prod_{i=p}^{n}\left[1-V\left(t_{i-1}, t_{i}\right)\right]^{-1} \Pi_{s}[1-V]^{-1} P\right| \\
& \quad \leqq \alpha\left(t_{0}\right)-\alpha\left(t_{n}\right) .
\end{aligned}
$$

For part (4) of Theorem II, suppose that $a>b, \beta$ is as in (iv), $\varepsilon>0$, and $P$ is in $D$. Since $M(\cdot, b) P$ is quasi continuous, $M([b, a], b) P$ is compact. Hence, there is a positive number $\delta$ such that if $Q$ is in $M([b, a], b) P, R$ is in $D$ such that $|Q-R|<\delta$, and $a \geqq x \geqq y \geqq b$ 
then $|V(x, y) Q-V(x, y) R| \leqq[\beta(x)-\beta(y)] \cdot \varepsilon / 3$. Suppose that $Q$ is in $D$ such that $|Q-P|<\delta,\left\{t_{p}\right\}_{0}^{n}$ is a subdivision of $\{x, y\}$ such that if $R$ is $P$ or $Q$ and $p$ is an integer in $[1, n]$ then

$$
\left|\prod_{i=p}^{n}\left[1-V\left(t_{i-1}, t_{i}\right)\right]^{-1} R-M\left(t_{p_{-1}}, b\right) R\right|<\delta .
$$

Then

$$
\begin{aligned}
& \left|\left\{\Pi_{t}[1-V]^{-1}-1\right\} P-\left\{\Pi_{t}[1-V]^{-1}-1\right\} Q\right| \\
& \quad \leqq \sum_{p=1}^{n}\left|V\left(t_{p-1}, t_{p}\right) \prod_{i=p}^{n}\left[1-V\left(t_{i-1}, t_{i}\right)\right]^{-1} P-V\left(t_{p-1}, t_{p}\right) \prod_{i=p}^{n}\left[1-V\left(t_{i-1}, t_{i}\right)\right]^{-1} Q\right| \\
& \quad \leqq[\beta(x)-\beta(y)] \varepsilon .
\end{aligned}
$$

Proof of Theorem III. This theorem established that the evolution operator $M$ which was found in Theorem II provides a solution to the initial value problem indicated in Theorem III. Note that the integral used is the Cauchy-left integral: If $f$ is a function from $[b, a]$ with values in $D$ then $(L) \int_{a}^{b} V[f]$ is approximated by $\sum_{p=1}^{n} V\left(t_{p_{-1}}, t_{p}\right) f\left(t_{p_{-1}}\right)$ where $t$ is a subdivision of $\{a, b\}$.

Lemma 3. Suppose that $a>b$ and $f$ is a function from $[b, a]$ to $D$ which is of bounded variation. It follows that $(L) \int_{a}^{b} V[f]$ exists; in fact, if $\varepsilon>0$ then there is a subdivision $s$ of $\{a, b\}$ such that if $\left\{t_{p}\right\}_{p=0}^{n}$ is a refinement of $s$ then

$$
\sum_{p=1}^{n}\left|V\left(t_{p-1}, t_{p}\right) f\left(t_{p-1}\right)-(L) \int_{t_{p-1}}^{t_{p}} V[f]\right|<\varepsilon .
$$

Lemma 4. Suppose that $b$ is in $S, P$ is in $D$, each of $f$ and $g$ is of bounded variation, and, for each $x \geqq b, f(x)=P+L \int_{x}^{b} V[f]$ and $g(x)=P+(L) \int_{x}^{b} V[g]$. It follows that if $x \geqq b$ then $f(x)=g(x)$.

Proof. With the supposition of the lemma, let $x$ be in $S$ such that $x \geqq b, \varepsilon$ be a positive number, and $\left\{t_{p}\right\}_{p=0}^{n}$ be a subdivision of $\{x, b\}$ such that

$$
\begin{aligned}
& \sum_{p=1}^{n}\left\{\left|\int_{t_{p-1}}^{t_{p}} V[f]-V\left(t_{p_{-1}}, t_{p}\right) f\left(t_{p_{-1}}\right)\right|\right. \\
& \left.\quad+\left|\int_{p_{-1}}^{t_{p}} V[g]-V\left(t_{p_{-1}}, t_{p}\right) g\left(t_{p_{-1}}\right)\right|\right\}<\varepsilon .
\end{aligned}
$$

Then 


$$
\begin{aligned}
\mid f(x)- & g(x)|\leqq| f(x)-g(x) \mid+\sum_{p=1}^{n}\left\{\mid\left[1-V\left(t_{p_{-1}}, t_{p}\right)\right] f\left(t_{p_{-1}}\right)\right. \\
& \left.-\left[1-V\left(t_{p_{-1}}, t_{p}\right)\right] g\left(t_{p_{-1}}\right)|-| f\left(t_{p_{-1}}\right)-g\left(t_{p_{-1}}\right) \mid\right\} \\
= & \sum_{p=1}^{n}\left\{-\left|f\left(t_{p}\right)-g\left(t_{p}\right)\right|+\mid\left[1-V\left(t_{p-1}, t_{p}\right)\right] f\left(t_{p_{-1}}\right)\right. \\
& \left.-\left[1-V\left(t_{p_{-1}}, t_{p}\right)\right] g\left(t_{p-1}\right) \mid\right\} \leqq \sum_{p=1}^{n}\left\{\left|\int_{t_{p-1}}^{t_{p}} V[f]-V\left(t_{p-1}, t_{p}\right) f\left(t_{p_{-1}}\right)\right|\right. \\
& \left.\left.\left.+\mid-\int_{t_{p-1}}^{t_{p}} V\right] g\right]+V\left(t_{p_{-1}}, t_{p}\right) g\left(t_{p_{-1}}\right) \mid\right\}<\varepsilon .
\end{aligned}
$$

Thus

$$
f(x)=g(x)
$$

Indication of proof for Theorem III. Suppose that $a>b, P$ is in $D$, and $s$ is a subdivision of $\{a, b\}$. Then

$$
\begin{aligned}
\mid \prod_{p=1}^{n} & {\left[1-V\left(s_{p_{-1}}, s_{p}\right)\right]^{-1} P-P-\sum_{p=1}^{n} V\left(s_{p_{-1}}, s_{p}\right) M\left(s_{p_{-1}}, b\right) P \mid } \\
= & \left|\sum_{p=1}^{n} V\left(s_{p_{-1}}, s_{p}\right) \prod_{i=p}^{n}\left[1-V\left(s_{i-1}, s_{i}\right)\right]^{-1} P-V\left(s_{p_{-1}}, s_{p}\right) M\left(s_{p_{-1}}, b\right) P\right| .
\end{aligned}
$$

Using the fact that $M([b, a], b) P$ is compact, together with the above corollary, we get that $M(a, b) P-P-(L) \int_{a}^{b} V M(\cdot, b) P=0$. Lemma 4 shows that this is the only solution to the Stieltjes integral equation.

ExAmple. Suppose that $g$ is an increasing, number valued function, $A$ is a function with values in a Banach space $G$, and that $A$ has the following properties: (Compare [6, p. 258].)

(a) If $t$ is a number then $A(t, \cdot)$ has domain all of $G$,

(b) If $P$ is in $G$ then $A(\cdot, P)$ is continuous,

(c) If $a>b, P$ is in $G$, and $\varepsilon>0$ then there is a positive number $\delta$ having the property that if $a \geqq u \geqq b$ and $Q$ is in $G$ such that $|Q-P|<\delta$ then $|A(u, Q)-A(u, P)|<\varepsilon$,

(d) If $a>b$ and $B$ is a bounded subset of $G$ then $A$ is bounded on $[b, a] \times B$, and

(e) If $t$ is a number, $P$ and $Q$ are in $G$, and $c>0$ then

$$
|[P-c A(t, P)]-[Q-c A(t, Q)]| \geqq|P-Q| \text {. }
$$

Also, as in $\left[6\right.$, p. 258] let $V(x, y) P=(L) \int_{y}^{x} d g A(, P)$ for $x \geqq y$ and $P$ in $G$.

Then $V$ is in $O A$ and if $c$ is a number and $P$ is in $G$ then the preceeding provides the only function $f$ such that

$$
f(x)=P-(L) \int_{x}^{c} d g A(, f) .
$$




\section{REFERENCES}

1. Carl W. Bitzer, Stieltjes-Volterra integral equations, Illinois J. Math., 14 (1970), 434-451.

2. W. P. Davis and J. A. Chatfield, Concerning product integrals and exponentials, Proc. Amer. Math. Soc., 25 (1970), 743-747.

3. Burrell W. Helton, Integral equations and product integrals, Pacific J. Math., 16 (1966), 297-322.

4. James V. Herod, Multiplicative inverses of solutions for Volterra-Stieltjes integral equations, Proc. Amer. Math. Soc., 22 (1969), 650-656.

5. — A Gronwall inequality for Linear Stieltjes integrals, Proc. Amer. Math. Soc., 23 (1969), 34-36.

6. - A pairing of a class of evolution systems with a class of generators, Trans. Amer. Math. Soc., 157 (1971), 247-260.

7. Coalescence of solutions for nonlinear Stieltjes equations, J. Reine Angew. Mathematik, 238 (1972), 100-104.

8. T. H. Hildebrandt, On systems of linear differentio-Stieltjes-integral equations, Illinois J. Math., 3 (1959), 352-373.

9. D. B. Hinton, A Stieltjes-Volterra integral equation theory, Canad. J. Math., 18 (1966), 314-331.

10. David Lowell Lovelady, A variation-of-parameters inequality, Proc. Amer. Math. Soc., 26 (1970), 598-602.

11. - Perturbations of solutions of Stieltjes integral equations, Trans. Amer. Math. Soc., 155 (1971), 175-187.

12. - Bounded solutions of Stieltjes integral equations, Proc. Amer. Math. Soc., 28 (1971), 127-133.

13. - Multiplicative integration of infinite products, Canad. J. Math., 23 (1971), 692-698.

14. - Addition in a class of nonlinear Stieltjes integrators, Israel J. Math., 10 (1971), 391-396.

15. J. S. Mac Nerney, Integral equations and semigroups, Illinois J. Math., 7 (1963), 148-173.

16. - A linear initial-value problem, Bull. Amer. Math. Soc., 69 (1963), $314-329$.

17. - A nonlinear integral operation, Illinois J. Math., 8 (1964), 621-638.

18. G. W. Marrah and T. G. Proctor, Solutions of some periodic Stieltjes integral equations, Proc. Amer. Math. Soc., 34 (1972), 121-127.

19. R. H. Martin, Jr., A bound for solutions of Volterra-Stieltjes integral equations, Proc. Amer. Math. Soc., 23 (1969), 506-512.

20. - Product integral approximations of solutions to linear operator equation, Proc. Amer. Math. Soc., 41 (1973), 506-512.

21. James A. Reneke, A product integral solution of a Stieltjes-Volterra integral equation, Proc. Amer. Math. Soc., 24 (1970), 621-626.

22. - Continuity for Stieltjes-Volterra integral equation, Technical Report, No. 41, Clemson University.

23. - Product integral solutions for hereditary systems, unpublished manuscript.

24. W. W. Schmaedeke and G. R. Sell, The Gronwall inequality for modified Stieltjes integrals, Proc. Amer. Math. Soc., 19 (1968), 1217-1222.

25. H. S. Wall, Concerning harmonic matrices, Arch. Math., 5 (1954), 160-167.

Received July 3, 1973.

Georgia Institute of Technology 



\section{PACIFIC JOURNAL OF MATHEMATICS}

\section{EDITORS}

RICHARD ARENS (Managing Editor)

University of California

Los Angeles, California 90024

R. A. Beaumont

University of Washington

Seattle, Washington 98105
J. DugundJI

Department of Mathematics

University of Southern California

Los Angeles, California 90007

D. Gilbarg and J. Milgram

Stanford University

Stanford, California 94305

\section{ASSOCIATE EDITORS}

E. F. BECKENBACH

B. H. NeUMANN

F. WOLF

K. YosHIDA

\section{SUPPORTING INSTITUTIONS}

UNIVERSITY OF BRITISH COLUMBIA

CALIFORNIA INSTITUTE OF TECHNOLOGY

UNIVERSITY OF CALIFORNIA

MONTANA STATE UNIVERSITY

UNIVERSITY OF NEVADA

NEW MEXICO STATE UNIVERSITY

OREGON STATE UNIVERSITY

UNIVERSITY OF OREGON

OSAKA UNIVERSITY
UNIVERSITY OF SOUTHERN CALIFORNIA

STANFORD UNIVERSITY

UNIVERSITY OF TOKYO

UNIVERSITY OF UTAH

WASHINGTON STATE UNIVERSITY

UNIVERSITY OF WASHINGTON

$\stackrel{*}{*} \stackrel{*}{*} \stackrel{*}{*}$ AMERICAN MATHEMATICAL SOCIETY




\section{Pacific Journal of Mathematics}

\section{Vol. 53, No. $1 \quad$ March, 1974}

Martin Bartelt, Strongly unique best approximates to a function on a set, and a finite

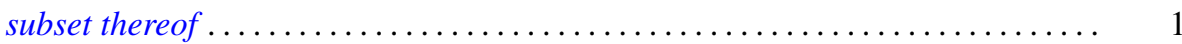

S. J. Bernau, Theorems of Korovkin type for $L_{p}$-spaces $\ldots \ldots \ldots \ldots \ldots \ldots \ldots \ldots \ldots$

S. J. Bernau and Howard E. Lacey, The range of a contractive projection on an

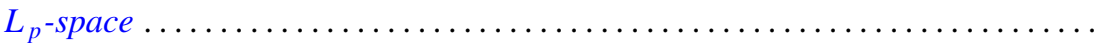

Marilyn Breen, Decomposition theorems for 3-convex subsets of the plane ......... Ronald Elroy Bruck, Jr., A common fixed point theorem for a commuting family of

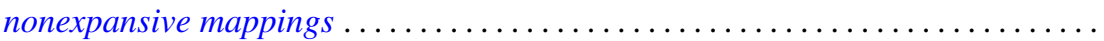

Aiden A. Bruen and J. C. Fisher, Blocking sets and complete $k$-arcs . . . . . . . 73

R. Creighton Buck, Approximation properties of vector valued functions . ......... 85

Mary Rodriguez Embry and Marvin Rosenblum, Spectra, tensor products, and

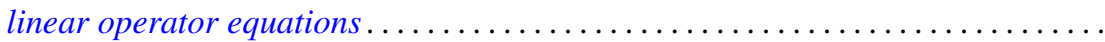

Edward William Formanek, Maximal quotient rings of group rings . . . . . . . . . 109

Barry J. Gardner, Some aspects of T-nilpotence . . . . . . . . . . . . . . . 117

Juan A. Gatica and William A. Kirk, A fixed point theorem for $k$-set-contractions

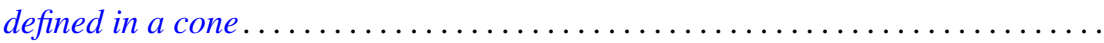

Kenneth R. Goodearl, Localization and splitting in hereditary noetherian prime

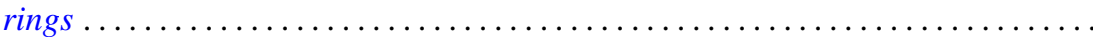

James Victor Herod, Generators for evolution systems with quasi continuous

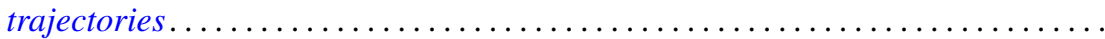

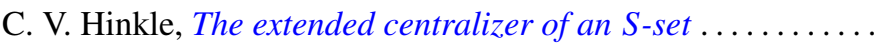

I. Martin (Irving) Isaacs, Lifting Brauer characters of p-solvable groups . . .

Bruce R. Johnson, Generalized Lerch zeta function ...........

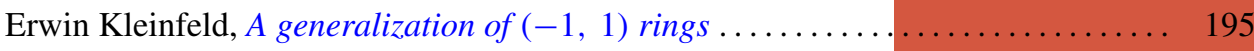

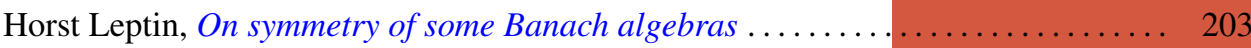

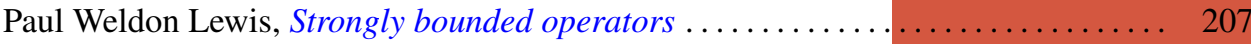

Arthur Larry Lieberman, Spectral distribution of the sum of self-adjoint

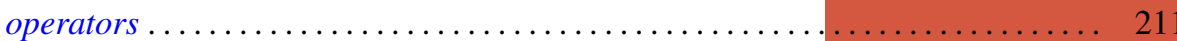

I. J. Maddox and Michael A. L. Willey, Continuous operators on paranormed spaces and matrix transformations

James Dolan Reid, On rings on groups ........................... 229

Richard Miles Schori and James Edward West, Hyperspaces of graphs are Hilbert cubes.

William H. Specht, A factorization theorem for p-constrained groups ...

Robert L Thele, Iterative techniques for approximation of fixed points of certain nonlinear mappings in Banach spaces ...............

Tim Eden Traynor, An elementary proof of the lifting theorem

Charles Irvin Vinsonhaler and William Jennings Wickless, Completely decomposable groups which admit only nilpotent multiplications .

Raymond O’Neil Wells, Jr, Comparison of de Rham and Dolbeault cohomology for

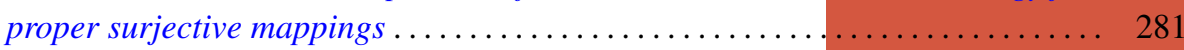

David Lee Wright, The non-minimality of induced central representations . . . . . 301 